\title{
Uwb Based Imaging Technique for Breast Tumour Detection by using Erar, Das and Dmas Algorithms
}

\author{
Ennam.Govinda, Vemuri. B.S.SrilathaIndira Dutt
}

\begin{abstract}
Breast tumor is high hazard infection among ladies which regularly causes perilous dread. So as to perceive the tumor tainted breast picture UWB imaging method is used. In this manner used breast picture is preprocessed for eliminating the noise and unnecessary information by stable wiener channel and Recursive least square channel. From this time preprocessed picture is given to FDTD for examining the breast picture for tumor acknowledgment. Accordingly got picture given to ERAR, $D A S$ and DMAS algorithms for removal of artifact from tumor speculated picture. In this manner acquired picture is made auto correlated for brightening up the tumor contaminated territories. At that point the breast pictures are segmented by edge-based tumor segmentation and the outcomes are characterized with DAS and DMAS algorithms for better accuracy and sensitivity of picture.
\end{abstract}

Keywords : Delay and Sum (DAS), UWB (Ultrawideband), Robust and Enhanced Artifact Resistant algorithm (ERAR), delay multiply and sum (DMAS).

\section{INTRODUCTION}

The most broadly perceived sickness among females is Breast Cancer and one of the primary wellsprings of death by and large [1]. But less normal in males, recognized event of breast cancer started lately.Early determination of breast malignant growth is one of the most testing and significant perspectives for the administration of the sickness, as it might be viable to distinguish the disease before it is spreading [2]. There are 3 consistently used screening processes for breast threatening development are Ultrasound (US), X-beam mammography and (MRI)Magnetic Resonance Imaging. Higher rate of fake positive results with US make it less useful than mammography, while MRI is for the most part prescribed to be used identified with mammography [3]. Despite the blessing of mammography, its desiresare clear: sensitivity is low [4], anguishing breast pressure, radiation introductionwith X-beams, which brings a potential risk of expanding malignant growth chance .Obstacles of present systems comprise annotion for better alternatives. Over the most recent couple of decades, various models of microwave imaging's are available in breast cancer detection, those are hybrid, active, passive methods. passive imaging frameworks attempt to recognize tumors reliant on heat contrasts among

\footnotetext{
Revised Manuscript Received on December 13, 2019.

* Correspondence Author

Ennam. Govinda*, Assoc. Professor,Dept. of ECE, Avanthi Institute of Engineering and Technology, Narsipatnam, Visakhapatnam, Andhra Pradesh, India. email: ennamgovinda19@gmail.com

Dr.Vemuri.B.S.SrilathaIndira Dutt, Department of ECE, GITAM University, Visakhapatnam, Andhra Pradesh, India. email: srilatha06.vemuri@gmail.com
}

typical, carcinogenic breast tumors with radiometer guide (6). Hybrid approaches separate typical tissues by the obvious acoustic waves transmitted from the thermoelastic extension when tissues are beneath enlightenment of microwaves.

The Active systems pick out normal and dangerous bosom tissues challenge to their big difference of dielectric residences at microwave frequencies [7]. Considering the entertainment procedure used, dynamic identification methods can be arranged into microwave tomography and (UWB)ultrawideband radar-based imaging. In microwave tomography, the spatial spreads of dielectric predictable and also conductivity inside the bosom are iteratively decided, thusly non-linear inverse scattering issues are incorporated.

The UWB radar methodologies, then again, sketch to apprehend the nearness and location of strong scatters, for instance, tumors, as opposed to quantitatively preparing the appropriation of dielectric properties. UWB radar-based imaging structures face a couple of troubles for bosom danger disclosure, two of them are antenna design, and the development of sensible (FDTD)finite difference time domain based [9] breast model.

Another troublesome test is picture arrangement calculation. The picture arrangement calculation is depended upon to give unmatched tumor recognizing confirmation limit, exact situating, strong robustness, and quick calculation speed. An assortment of picture arrangement calculations has been proposed over 10 years prior. Picture development calculations are assembled into data dependent and data independent calculations. Information subordinate calculations can imitate high goals pictures when cluster guiding vector identifying with the signal (SOI)of interest is unequivocally known, which is troublesome reasonable

bosom imaging circumstances. The data independent algorithms are free from this earlier data, and be continually created. Various data independent algorithms are proposed starting late are (DAS)delay and sum, delay multiply and sum (DMAS) and Enhanced robust Artifact Resistance algorithm (ERAR). Compared with DAS, DMAS, ERAR calculation have improved execution of clutter rejection, capacity of distinguishing numerous dissipates in homogenous and heterogenous breast. Where the presence of glandular tissue is considered, due to the presence of glandular tissue gives lot of attenuation, scattering of backscattered signals, making it increasing hard, to recognize if any little tumours present. 


\section{Uwb Based Imaging Technique for Breast Tumour Detection by using Erar, Das and Dmas Algorithms}

In these paper correlations of DAS, DMAS, and ERAR image formation algorithms are recognizing and qualities of these algorithms are inspected under different situations: homogenous and heterogenous breast models with varied densities.

\section{RELATED WORK}

Recognition and classification of breast tumor in the absence of human intervention needs a vast analysis and it include multiple number of complications. Numerous researchers have looked into through this territory fortumor recognition.in this area numerous works regarding tumor analysis discussed and evaluated. AsawariK. Chinchanikar*, Dr. R. S. Kawitkar**(10) ; proposed a methodology for generation and transmission of UWB signal and testing the performance with S-V channel.

Yusnita Rahayu1, Tharek Abd. Rahman1, Razali Ngah1 (11) written the paper on applications of ultrawideband signals in medical fields. Jack. A. Leendertz,Maciej Klemm(12) An improved antenna array was proposed for radar-based breast malignant growth imaging. The improvement was cultivated by growing the amount of gathering contraptions in the bunch to 31 segments, similarly as by improving the radio wire structure itself. Using an exploratory course of action, with homogeneous curved breast phantoms, we have displayed liberal imaging improvement with the new radio wire bunch. The new system is similarly prepared to recognize up to $7 \mathrm{~mm}$ tumor apparitions in any region inside the breast, indeed, even as close as $4 \mathrm{~mm}$ from the skin layer. In this DAS calculation is utilized. A.F. Mirza, F. Abdulsalam(13).proposed a perfect ultra wideband application for identifying tumor malignant growth inside breast tissues utilizing a FDTD numerical strategy. 1D, 2D and 3D FDTD models are researched tentatively and the most ideal methods for distinguishing the presence of harmful tissue are talked about. pulse with two bandwidth of fourGH,targeted at $6 \mathrm{GHz}$, had been utilized for excitation, and their appearance from tumor counterparts of specific positions and sizes have beenplotted and recorded. But this algorithm failed underneath severally dense breasts.

Falah H.Ali,seniormember,IEEE(14);proposed method of Adaptive Combining via Correlation Exploration (ACE) algorithm for breast illness distinguishing proof, It examines and mishandles the connection between back dissipated signals and close by comprehension reference signals made inside each get-together of neighboring getting receiving wires. High relationship signals are additively picked, added and weighted by the consequence of their contrasting coefficients, molding the intensity of each pixel inside an imaging an area. The sufficiency of proposed estimation is endorsed on 3D accurate finite difference time domain (FDTD) breast model. ACE wearing down homogeneous and heterogeneous, inadequate and sensibly thick bosom models, joined with perfect, pragmatic artifact removal techniques.ACE algorithmexhibitssuperior performance thanDMAS and DAS algorithms.
Tengfei Yin, Student Member of IEEE (15); composed a paper on Robust and Artifact Resistant (RAR) calculation. ItaffordsRobust and Artifact Resistant (RAR) calculation, in thata local pair wise correlation-based weighting is intended to beat the unfavorable impacts from both curio and glandular tumors. In RAR, backscattered alerts are time-moved, added and weighted by the most extreme mix of the neighboring pair wiserelationship coefficients between moved sign, shaping the intensity of every point inside an imaging region. This RAR is appropriate for the beginning time malignancy location in low to medium thickness breasts.

E.Govinda,V.B.S.S Indira Dutt, Senior member, IEEE(16) proposed method of Hybrid UWB Based Image technique for breast tumour detection by using Enhanced Robust and Artifact Removal Algorithm. This work develops a unique methodology whereas the UWB image is pre-processed using Adaptive wiener filter for noise removal and Recursive least square filter for eliminating unnecessary data. Thus,

pre-processed image is fed to FDTD for frequency \& time domain for tumour response. Thus, obtained images are processed with RAR algorithm in which backscattered images are time shifted, weighted and summed. These summed images are segmented by edge-based tumour

recognition for breast tumour identification. The segmented images are auto correlated for brightening tumour area and enhanced for further classification using DAS \& DMAS. Whereas DMAS fed tumour images have high accuracy and sensitivity.

\section{PROPOSED WORK}

In the created approach acknowledgment and separation of tumorous breast is finished by using UWB imaging framework. Along these lines, picked up UWB pictures are pre-processed by using Adaptive wiener channel for denoising the picture and Recursive least square channel is utilized for wiping out superfluous information in breast image. The input image requires FDTD for electromagnet signal to enter into the breast tissues for acknowledgment of tumors. For precise distinguishing proof of tumor in the breast tissues different image formation algorithms is created and UWB image is handled. In this manner, handled image experiences auto correlation for lighting up the influenced zones. At that point segmentation is finished by edge-basedsegmentation technique and afterward the tumor areas found with high exactness and every one of the outcomes are compared among the algorithms for example DAS, DMAS and ERAR for further characterization.

\section{METHODOLOGY}

UWB images are the information pictures which are pre-processed by utilizing two distinct channels for remove of noise and undesirable information. Thus, acquired image is handled by FDTD for penetration of signal into tissues of breast. For tumor distinguishing proof ERAR algorithm is used and images are in this way lit up via auto correlation and segmented by edge-based segmentation with high sensitivity and grouped by DAS and DMAS algorithms. 
i/p image Pre-processing

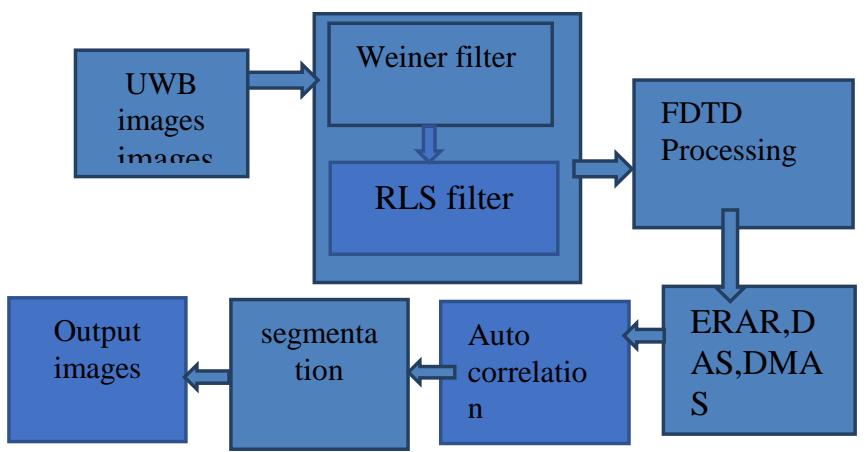

Figure. 1. Block diagram of the breast cancer detection system

\section{IMAGE PROCESSING}

Ultra-Wide Band (UWB) is a huge imaging method which defeats every one of the deficiencies of other imaging system. UWB is fit for accomplishing most extreme information rates up to $1 \mathrm{Gbps}$. UWB uses pulses with minimum power. UWB pulses have highest resolution $\mathrm{s}$ with various ways for tumor location. Need for UWB imaging

1. Remarkable differentiation in-between dielectric properties of ordinary and irregular breast tissues

2. Disposes of pressure of breasts tissues which isn't the reason for tumor.

3. Starting acknowledgment and restriction tumor cells in breast.

\section{PREPROCESSING}

In image processing, picture acquisition and pre-processing are the rudimentary requirement for tumor detection. In this work for pre-processing wiener channel and Recursive channel is used. Adaptive wiener channel is a recognizable procedure used for enhancement of images.

Essential function of adaptive wiener channel is to eliminates the additive noise in UWB image of breast. It expels frequent deblurring. Adaptive wiener filter assumes an ideal job in limiting restricting the mean squared deviation or error. Channel constrains the absolute mean square deviation in reverse sifting and smoothening of noises. The least mean square deviation point responds to the diminished error control. At this working point the mean square deviation surface has gradient value is zero and channel weight vector has its optimal worth. A signal is absolutely recoverable from noises when the spectra of the signal and noise don't cover each other. In case the signal and noise possess various pieces of the recurrence range, they can be isolated by utilizing either low pass or high pass channel. In case the signal and noise has cover spectra, for this circumstance it is ridiculous to thoroughly segregate the sign from clamour yet the effects of commotion can be lessened by using wiener channel. It disposes of the noisy signal and required signal is surveyed by $\hat{S}(n)=\sum_{K=0}^{p-1} w_{k} x(n-K)$

$\hat{S}(n)=w^{T} x$

Where $x(n)=$ input sequence

$w_{k}=$ filter coefficients

$\hat{S}(n)=$ evaluated output sequence

The residual error $\mathrm{e}(\mathrm{n})=\hat{S}(n)-s(n)$

Thus, obtained denoised image from Adaptive wiener filter is fed to Recursive least filter for eliminating unnecessary data in the image. To eliminate the artifact Recursive least square (RSL) filter is utilized.

Recursive least filter for eliminating unnecessary data in the image.

If $d(n)$ is the signaltransmitted over an noisy channel that causes it to be received as

$$
\mathrm{X}(\mathrm{n})=\mathrm{v}(\mathrm{n})+\sum_{K=0}^{q} b_{n}(k) d(n-K)
$$

*additive noise is $\mathrm{v}(\mathrm{n})$.

Main aim of RLSF is to recover d(n) with filter coefficients "w".i.e

$$
\mathrm{k} \quad \mathrm{d}(n)=\sum_{K=0}^{p-1} w_{k} x(n-K)
$$

Signals back scattered constitutes of two types. Initial and final stages Initial stage constitutes incident signals from interface skin fat. In final stage tumour response fatty tissue response are found.

Let $\mu_{x}$ be the $l * N$ vector with $N$ times of samples with desired signal $x$ and

$\mathrm{u}=\left[\mathrm{u}_{\mathrm{k}+1}+\mathrm{u}_{\mathrm{k}+2}+\ldots+\mathrm{u}_{\mathrm{k}+\mathrm{m}}\right]^{\mathrm{T}}$

processed signals, $\mathrm{w}(\mathrm{n})=\left[\mathrm{w}_{\mathrm{X}+1}(\mathrm{n}), \mathrm{w}_{\mathrm{W}+2}(\mathrm{n}), \ldots \ldots \ldots \mathrm{w}_{\mathrm{X}+\mathrm{m}}(\mathrm{n})\right]^{\mathrm{T}}$

Thus, the image is processed by eliminating unwanted noise and data is fed to FDTD processing.

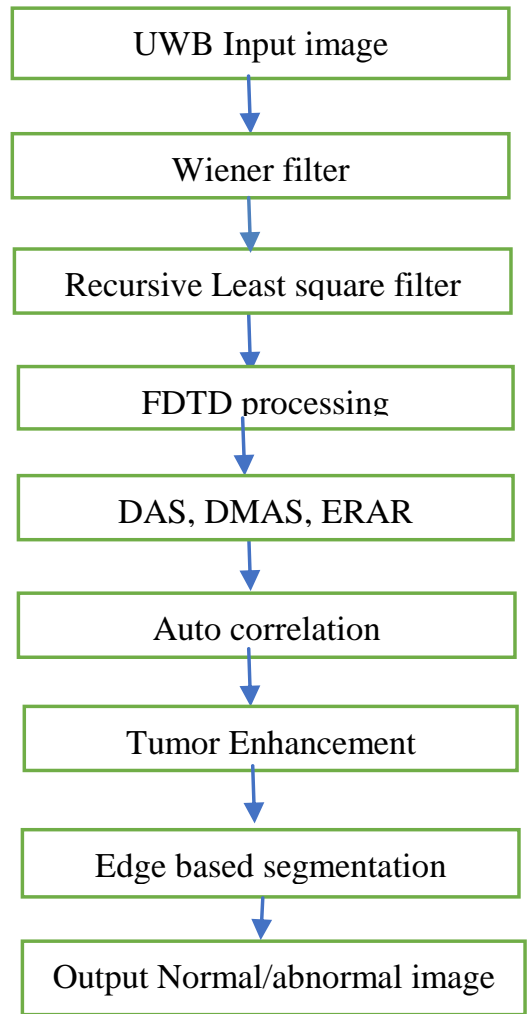

Fig. 2. Flowchart of proposed system 


\section{FREQUENCY DOMAIN/TIME DOMAIN PROCESSING (FDTD)}

Every single imaging procedure needs high accuracy in breast pictures for scattering and penetration of UWBsignals within the breast. The technique ofof frequency domain \& time domain is a numerical strategy for execution of electromagnetic (EM) signals into the tissues of breast. This EM signal creates heat in the breast tissues [8]. The amount of temperature increments as for introduction limitation to fields,the specific energy absorption rate is in between $100 \mathrm{KHz}$ to $10 \mathrm{GHz}$ is [20].

Specific energy absorption rate $\mathrm{SAR}=\frac{\sigma] \mathrm{E}]^{2}}{\rho}$

$\rho=$ density

$\sigma=$ conductivity

Speculation dependent on the FDTD strategy is essential. To handle an electromagnetic issue, the idea is to simply discretize, both in presence, the Maxwell's conditions with central difference approximations.Maxwell's conditions can be composed as

$$
\begin{aligned}
& \partial \mathrm{E} / \partial \mathrm{t}=1 / €_{0} \nabla \times \mathrm{H} . \\
& \partial \mathrm{H} / \partial \mathrm{t}=1 / \mu_{0} \nabla \times \mathrm{E} .
\end{aligned}
$$

The Maxwell curl equation by FDTD.

$$
\begin{aligned}
& \text { 圆 } X H=\sigma E+\epsilon \partial E / \partial t \\
& \text { 固 } X E=-\mu \partial H / \partial t
\end{aligned}
$$

Where ' $E$ ' is Electric lines of force

' $\mathrm{H}$ ' ismagnetic flux

$\mu$ is permeability

Thus, the permittivity, permeability and sensitivity of image are improved by this technique. Thus, processed UWB breast images from FDTD id fed to the image formation algorithms for recognition tumour.

\section{DELAY AND SUM (DAS)ALGORITHM}

The DAS [5] are commonplace strategy. After segmentation the specific time delays are determined for every one of the signals got. For computation of the time delay of transmitting and receiving antenna the position of antenna is dissected. In all the area every shifted time responses are summed and integrated. Windowed signal performs integration. Reconciliation window length is picked concerning data transfer capacity of the framework.

$F_{i}(a, b, c)=\int_{0}^{\pi} \sum_{i=1}^{M} w_{i}(a, b, c) \cdot y_{i}\left(t-T_{i}(a, b, c)\right)^{2} d t$

Where $M=\frac{N(N+1)}{2}$

$T_{\mathrm{i}}=$ Time delay

$y_{i}=$ Radar signal

$\tau=$ Integration window length

\section{DELAY MULTIPLY AND SUM(DMAS)ALGORITHM}

By using DMASalgorithm calculate all the picture focuses ascertain time delay for all cluster components are connected and multiplied and the similarity is determined. DMAS is created to conquer the deficiencies of DAS calculation. As DAS has insignificant resolution of breast pictures and contributes exceptionally to off axis signals. Accordingly, the segmented breast images are assessed by DAS and DMAS while DMAS furnishes tumor acknowledgment and classification with high accuracy and execution

\section{ENHANCED ROBUST AND ARTIFACT RESISTANT (ERAR) ALGORITHM}

The created work a novel alternate way and Artifact Resistant Algorithm is proposed to acknowledgment of tumor with high precision. At the outset the layer of skin is the first scattered in which EM signal comprises enormous extent subsequently dissipating is stronger than latter time response [19]. The skin is enormous permittivity than greasy tissues. Henceforth diminutions of artifact signals have to be done. Acknowledge of beginning stage artifact must be undeniably dispensed with as for earlier data managed from typical breast pictures.

The $i$ th handset from perfect tumor reaction in discrete structure showed as

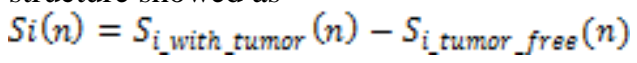

Where $n=1,2, \ldots, K$

$S_{\text {i with tumor }}(n)=$ the received backscattersignal at $i$ th handset from tumour.

$S_{i_{i} \text { tumor } \text { fres }_{1}(n)}=$ the received backscatter signal at same handset from breast model without tumour

Elimination of artifact have to be achieved if not, the tumour intensity and size cannot be recognized. Hence RAR algorithm is developed for evaluating the intensity of all the pixels.

Let $l$ denote the $l$ th location of pixel in the image. For all the location correlation between time shifted signals gets exploited by RAR. Hence time shifted signal has maximum correlation in between response of tumour cells.

For evaluating the intensity values of $l$ the following steps are evaluated.

Step 1: With respect to corresponding time delay at $l$ all pre-processed $S i(n)$ is time shifted. Signal time shifted are exhibited as $S_{i}\left(n+\tau_{\text {in }}\right)$

Antenna number is denoted as $A$. After eliminating artifact signal with tumor is gathered. Henceforth for each location $l$ there are time delays with $A$ set with respect to transceiver. Let $\operatorname{Sum}_{1(n)}(n=1,2, \ldots \ldots, k)$ represent every signal with time shift.

$S u m_{1(n)}=\sum_{i=1}^{A} S_{i}\left(n+\tau_{i n}\right)$

Step 2: Tumor reaction is expanded and antagonistic impacts came about at beginning and last stages artifact, a weight factor wf_1 for $l$ th area is given. The coefficient for Pearsons correlation is assessed as

$$
\begin{aligned}
& \mathrm{r}_{\mathrm{ij}-1}=\frac{\sum_{\mathrm{n}=1} X_{\mathrm{i}}(\mathrm{n}) \mathrm{X}_{\mathrm{j}}(\mathrm{n})}{\sqrt{\sum_{\mathrm{n}=1}^{\mathrm{m}} \mathrm{X}_{\mathrm{i}}(\mathrm{n})^{2} \sum_{\mathrm{n}=1}^{\mathrm{s}} \mathrm{X}_{\mathrm{j}}(\mathrm{n})^{2}}} \\
& \text { Where } X_{\mathrm{i}}(n)=S_{\mathrm{i}}\left(n+\tau_{\mathrm{in}}\right) \\
& X_{j}(n)=S_{j}\left(n+\tau_{\mathrm{in}}\right), i=i+1
\end{aligned}
$$

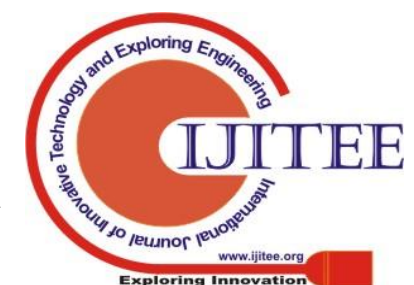


Step 3: For evaluating t $l$ th location intensity Additionally aligned time signals don't respond to location of tumor in complex medium. The sum has high an incentive at area of tumor. The constituted signal $c_{1}(n)$ is

$$
C_{1}(n)=w f_{1} \cdot \operatorname{Sum}_{1}(n)
$$

For all the location the above steps are repeated inside the region of image.

$$
I_{1}=\sum_{n=1}^{m}\left[C_{1}(n)\right]^{2}
$$

Thus, the Artifact is removed by utilizing RAR algorithm from breast image with tumor.

\section{Auto correlation:}

For enhancing or brightening a specific area in a UWB image Auto correlation is performed with the tumorous image itself as a function offset or log. Auto correlation is represented by Fourier transforms

$$
f(x, y)=\int_{-\infty}^{\infty} \int_{-\infty}^{\infty \infty} f\left(x^{1}, y^{1}\right) \cdot f\left(x+x^{1}, y+y^{1}\right) d x^{1} d y^{1}
$$

Where $f(x, y)=$ function which brightens image

Thus obtained brightened image of breast tumor cells are fed to enhancement of image by eliminating the artifact and the image is enhanced.

\section{Edge based segmentation}

segmentation is a procedure subdividing a picture into areas. In this work edge-based division is used for acknowledgment of tumor in breast picture with high precision. The undesirable pixel in the edge of the picture is fragmented with the high force. Subsequently upgraded picture is divided with tumor acknowledgment.

\section{RESULTS}

The created work is exhibited on MATLAB programming tool kit with 4Gb RAM. For assessing the proposed work eight input UWB images (8) are used for ascertaining the force of tumor. The info pictures are breaking down by different advances. It incorporates pre-pre-processing, Frequency Domain/Time Domain processing, DAS, MDAS and ERAR calculations, Tumor enhancement, segmentation and classification of tumour images.

In pre-processing Adaptive wiener channel and recursive channel for evacuation of superfluous noise and information in the UWB breast picture. At that point the picture is encouraged to FDTD and artifact removal. In this segment performance of the DAS, DMAS, and ERAR calculations is assessed for acknowledgment of tumor images. In this manner, got breast image with tumor is assessed by separating the tumor image with DAS, DMAS. By using DAS and DMAS the tumor contaminated breast picture is arranged with conductivity, specificity.

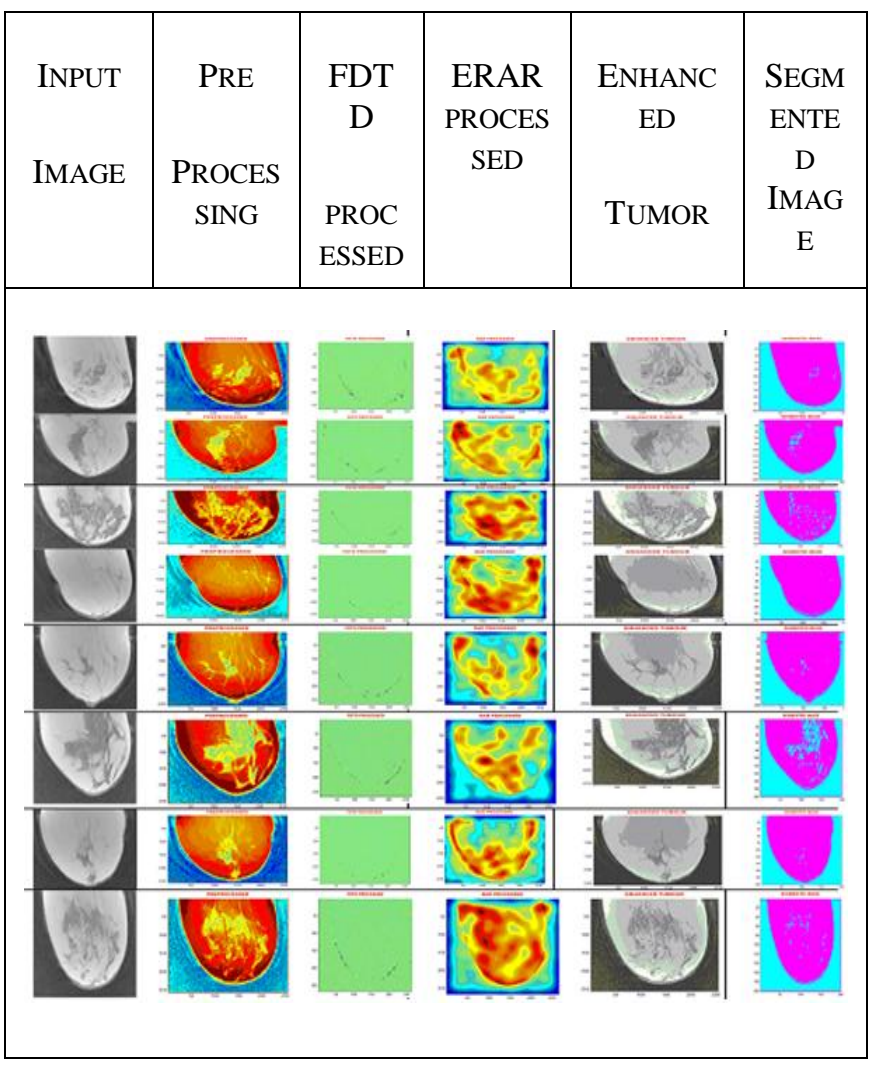

Fig. 3. various stages of tumour recognition

Delay and sum (DAS) are a familiar methodology. After segmentation the exact time delays are calculated for all the signals received. For calculation of the time delay of transmitting \& receiving antenna the position of antenna is analysed.

In all the location every shifted time response is summed and integrated. Windowed signal performs integration. Integration window length is chosen with respect to bandwidth of the system.

$$
\begin{aligned}
& F_{\theta}(a, b, c)=\int_{0}^{\pi} \sum_{i=1}^{M} w_{i}(a, b, c) \cdot y_{i}\left(t-T_{i}(a, b, c)\right)^{2} d t \\
& \text { Where } M=\frac{\mathbb{N}[N+1)}{2} \\
& T_{i}=\text { Time delay } \\
& \quad y_{i}=\text { Radar signal } \\
& \tau=\text { Integration window length }
\end{aligned}
$$

In DMAS algorithm all the image points calculate time delay for all array elements are linked and multiplied and similarity is calculated. DMAS is developed to overcome the shortcomings in DAS algorithm. As DAS has minimal resolution of breast images and contributes highly to off axis signals. Thus, the segmented breast images are evaluated by DAS and DMAS whereas DMAS provides tumour recognition and classification with high accuracy and performance. 
Uwb Based Imaging Technique for Breast Tumour Detection by using Erar, Das and Dmas Algorithms

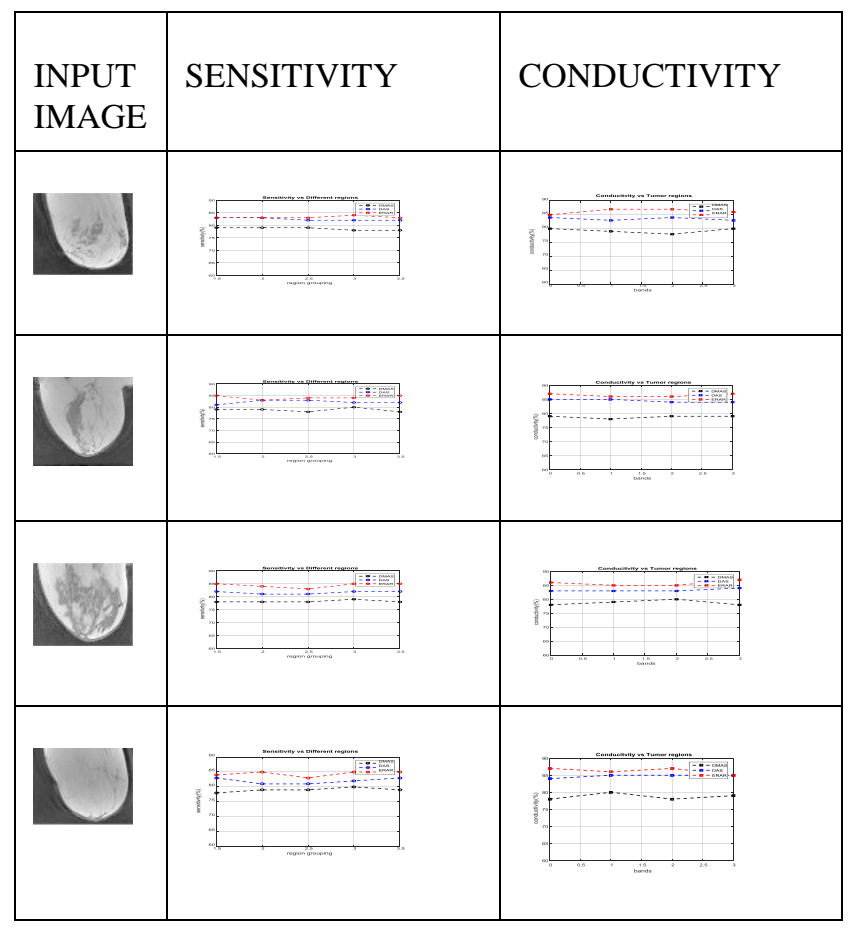

Fig. 4. Representation of sensitivity and conductivity of tumour images

The above figure 4 shows the input images with tumour and the tumour infected images are differentiated by evaluating the specificity and conductivity of the images. These factors are evaluated by utilizing the DAS, DMASand ERAR algorithm. Thus, classified breast images are exhibited in the figure. From the above figure it is observed that the DMAS outperforms the DAS and ERAR and produces tumour infected image with high accuracy and sensitivity.

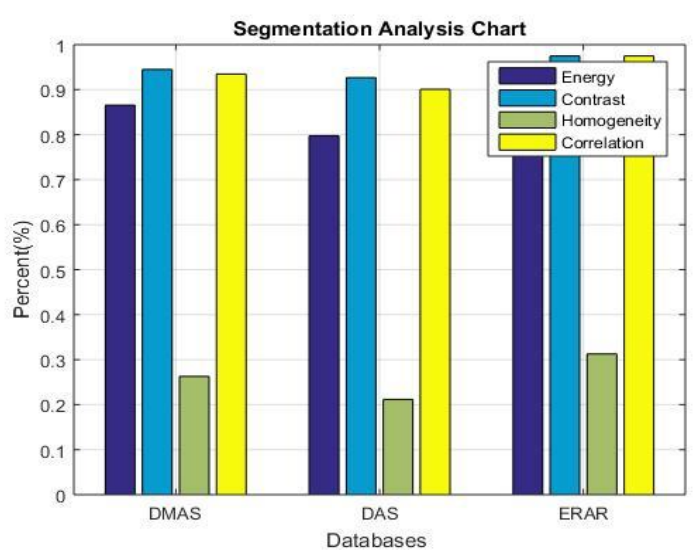

Fig. 5. Segmentation Analysis

\section{MSE and PSNR}

The mean square error MSE represents the cumulative squared error between enhanced and original image.

$$
\text { MSE }=\sum_{u, v}(I 1(u, v)-I 2(u, v))^{2} / \mathrm{u} \times \mathrm{v}
$$

Peak signal-to-noise ratio (PSNR) represents the maximum pixel value of image to mean squared deviation.

$\mathrm{PSNR}=10 \log _{10}\left(\mathrm{R}^{2} / \mathrm{MSE}\right)$

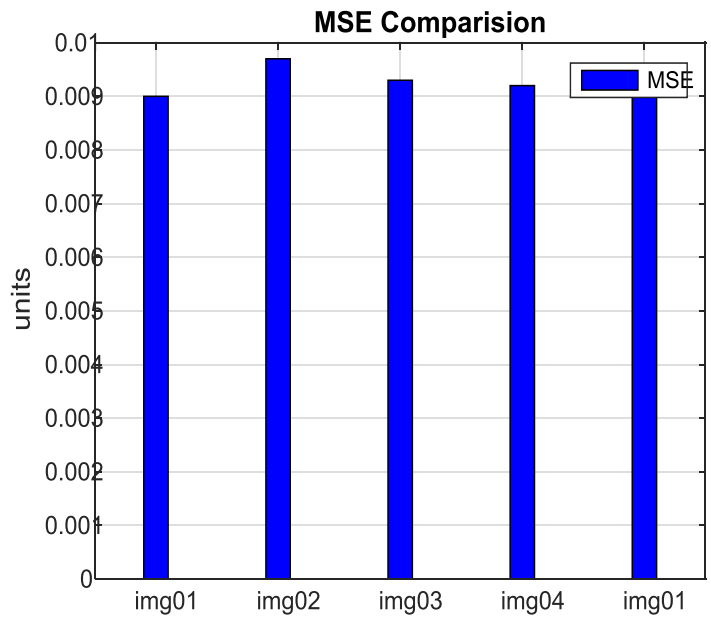

Fig. 6. MSE Comparison PSNR Comparision

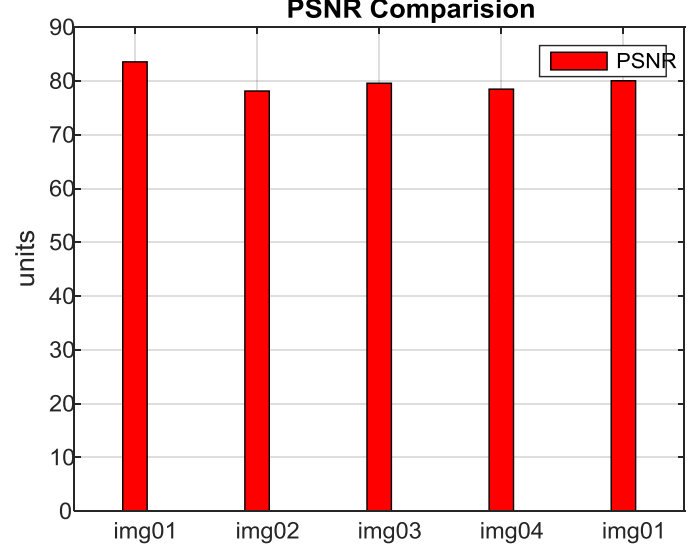

Fig.7. PSNR Comparison

\section{CONCLUSION}

The created strategy for acknowledgment of breast tumor is distant from everyone else through UWB imaging method. This work builds up a one of a kind system though the UWB images is pre-processed utilizing Adaptive wiener channel for noise removal and Recursive least square channel for disposing of pointless information. In this manner, pre-pre-processedimage is fed to FDTD for frequency and time domain for tumor response. In this way, got pictures are processed with DAS, DMAS, and ERAR algorithms in which backscattered images are time shifted, weighted and summed. These summedimages are segmented by edge-based tumor acknowledgment for breast tumor recognizable proof. The divided pictures are auto correlated for lighting up tumor territory. at last, ERAR encouraged tumor pictures have high accuracy and sensitivity.

\section{REFERENCES:}

1. Bray F, Center MM, Ferlay J, Ward E, Forman D, a cancer journal for clinicians - 2011, Mar-Apr; 61(2): 69-90. Doi 10.3322/caac.20107. Epub 2011 Feb 4.' Global cancer statistics.

2. J. Luttges and I.Schreer "Breast cancer early detection", Radiologic Pathologic Correlations from head to toe. PP No. 767-784, Springer 2005. 
3. Katrina Armstrong, Constance D.Lehman and Suzanne W.Fletcher " Screening for Breast Cancer" JAMA: journal of the American Medical Association, 2005 Mar 9, 293 (10) 1245-1256, doi: 10.1001/jama.293.10.1245.

4. Dr Carla Boetes, Dr Wylie Burke, Dr Steven Harms and Dr Martin O.Leach "American Cancer Society Guidelines for Breast Screening with MRI as an Adjunct to Mammography" First published: 19 February 2009, 1414.

5. M.Klemm,I.J.Craddock,J.A.Leendertz,A.Preece,andR.Benjamin "Improved delay-and-sum beamforming algorithm for breast cancer detection," International Journal of Antennas and Propagation, Vol.3, 2008, pp.1-9

6. Anders Mattsson, Erik Holmberg, Roy Shore "Radiation Effects on Breast Cancer Risk: A Pooled Analysis of Eight Cohorts" Radiation Research: August 2002, Volume 158, No.2, PP-220-235.

7. Allen Taflove and Jack E.Bridges "Two-Dimensional FDTD Analysis of a Pulsed Microwave Confocal System for Breast Cancer Detection: Fixed-focus and Antenna-Array Sensors" IEEE Transactions on biomedical engineering, Volume 45, No. 12, December 1998.

8. The UWCEM Numerical Breast Phantom in Lazebnik et al,PhysicsMedicalBiol.2007, http://uwcem.ece.wise.edu/MRIdatabase/index.html

9. The Finite-Difference Time-Domain Method, 3rd, MA: Artech House, 2000, ch. 7-9, pp. 273-406.A. Taflove, and S. C. Hagness, Computational electrodynamics:

10. Dr.R.S. Kawitkar,AsawariK.Chinchanikar,Performance Analysis of UWB System; International Journal of Scientific and Research Publications, Volume 2, Issue 9, September 20121 ISSN 2250-3153.

11. Ultra Wideband Technology and Its Applications Yusnita Rahayu1, Tharek Abd. Rahman1, Razali Ngah1, P.S. Hall2; 978-1-4244-1980-7/08/\$25.00 @2008 IEEE.

12. Maciej Klemm, Jack. A. Leendertz, David Gibbins, Ian J. Craddock, Alan Preece, and Ralph Benjamin; Microwave Radar-Based Differential Breast CancerImaging: Imaging in Homogeneous Breast Phantoms and Low Contrast Scenarios; IEEEtransactions on antennas and propagation, vol. 58 , no. 7, july 2010.

13. A.F. Mirza, F. Abdulsalam, R. Asif, Y.A.S. Dama, M. M. Abusitta, F. Elmegri, R.A. Abd-Alhameed,J.M.Noras, R. Qahwaji 1School of Engineering and Informatics, University of Bradford, UK; Breast Cancer Detection using 1D, 2D and 3D FDTD Numerical Methods; 2015 IEEE International Conference.

14. Tengfei Yin, Student Member, IEEE, and Falah H. Ali*, Senior Member, IEEE;Adaptive Combining via Correlation Exploration for Ultrawideband Breast Cancer Imaging; 1536-1225 (c) 2013 IEEE

15. FalahH.Ali,senior Member,IEEE and Constantino Carlos Reyes Aldasoro,seniorMember,IEEE; A Robust and Artifact Resistant Algorithm of UWB system for Breast Cancer detection;0018-9294@ 2015 IEEE.

16. V.B.S.SIndiraDutt,Seniormember,IEEE,E.Govinda,member,IEEE(27) ; Hybrid UWB Based Image technique for breast tumour detection by using Enhanced Robust and Artifact Removal Algorithm;2277-3878 Volume-7,Issue-6,IJRTE ,March 2019.

\section{AUTHORS PROFILE}

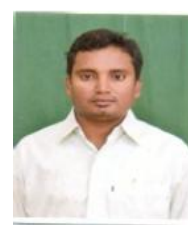

Ennam Govinda has completed his M.Tech in DSCE from JNTU Hyderabad. He obtained his B.Tech degree in ECE from V.R Siddharthaengineering college,BZA, AndhraPradesh. He took his current position as associate professor of ECE in Avanthi engineering college, Visakhapatnam. In addition to this he is doing Ph.D. at GITAM University, Visakhapatnam, A.P, India.

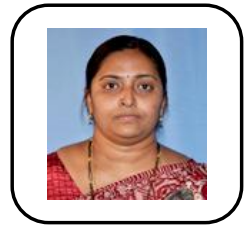

Dr.Vemuri.B.S.Srilatha Indira Duttpresently working as professor in Gitam University in ECE department, Visakhapatnam. She has done her B.Techfrom V.R. Siddhartha engineering college in ECE and Masters from Andhra University. She received her Ph.D. from Andhra Pradesh,India. With more than 18 years of experience she is an expert faculty member in microwave and radars. More than 24 research papers were published by her in various International and National conferences. She is a life member in various professional bodies like ISTE, CSI, IEEE 